EPJ Web of Conferences 113,05025 (2016)

DOI: $10.1051 /$ epjconf/201611305025

(C) Owned by the authors, published by EDP Sciences, 2016

\title{
Strong diquark correlations inside the proton
}

\author{
Jorge Segovia ${ }^{1, a}$ \\ ${ }^{1}$ Instituto Universitario de Física Fundamental y Matemáticas (IUFFyM), \\ Universidad de Salamanca, E-37008 Salamanca, Spain
}

\begin{abstract}
Quantum Chromodynamics is thought to be the relativistic quantum field theory that describes the strong interaction of the Standard Model. This interaction produces mesons but it is also able to generate quark-quark (diquark) correlations inside baryons. In this work, we employ a continuum approach to QCD based on Dyson-Schwinger equations to calculate the electromagnetic form factors of the proton and analyze in a deeper way the consequences of having strong diquark correlations. Comparison with the experimental data reveals that the presence of strong diquark correlations within the proton is sufficient to understand empirical extractions of the flavour-separated form factors. The explained reduction of the ratios $F_{1}^{d} / F_{1}^{u}$ and $F_{2}^{d} / F_{2}^{u}$ at high $Q^{2}$ in the quark-diquark picture are responsible of the precocious scaling of the $F_{2}^{p} / F_{1}^{p}$ observed experimentally.
\end{abstract}

\section{Origin of diquarks}

Quantum Chromodynamics (QCD) is the strong-interaction part of the Standard Model of Particle Physics and solving QCD presents a fundamental problem that is unique in the history of science. Never before we have been confronted by a theory whose elementary excitations are not those degrees of freedom readily accessible via experiment, that is, whose elementary excitations are confined. Moreover, QCD generates forces which are so strong that they produce about $98 \%$ of the mass of the proton and, consequently, most of the mass in the visible Universe. The underlying phenomenon which generates this mass - from nothing - is dynamical chiral symmetry breaking (DCSB). Neither confinement nor DCSB are apparent in QCD's Lagrangian and yet they play the dominant role in determining the observable characteristics of real-world QCD, e.g., hadron masses and coupling.

An important consequence of DCSB is that any interaction capable of creating bound-states of a light dressed-quark and -antiquark will necessarily also generate strong colour-antitriplet correlations between any two dressed quarks contained within a nucleon. Although a rigorous proof within QCD cannot be claimed, this assertion is based upon an accumulated body of evidence, gathered in two decades of studying two- and three-body bound-state problems in hadron physics (see, for instance, Refs. [1-5]). No realistic counter examples are known; and the existence of such diquark correlations is also supported by simulations of lattice QCD $[6,7]$.

The properties of diquark correlations have been charted. Diquarks are confined. Additionally, owing to properties of charge-conjugation, a diquark with spin-parity $J^{P}$ may be viewed as a partner to the analogous $J^{-P}$ meson [1]. It follows that scalar, isospin-zero and pseudovector, isospin-one diquark correlations are the strongest; and whilst no pole-mass exists, the following mass-scales,

\footnotetext{
ae-mail: segonza@usal.es
} 

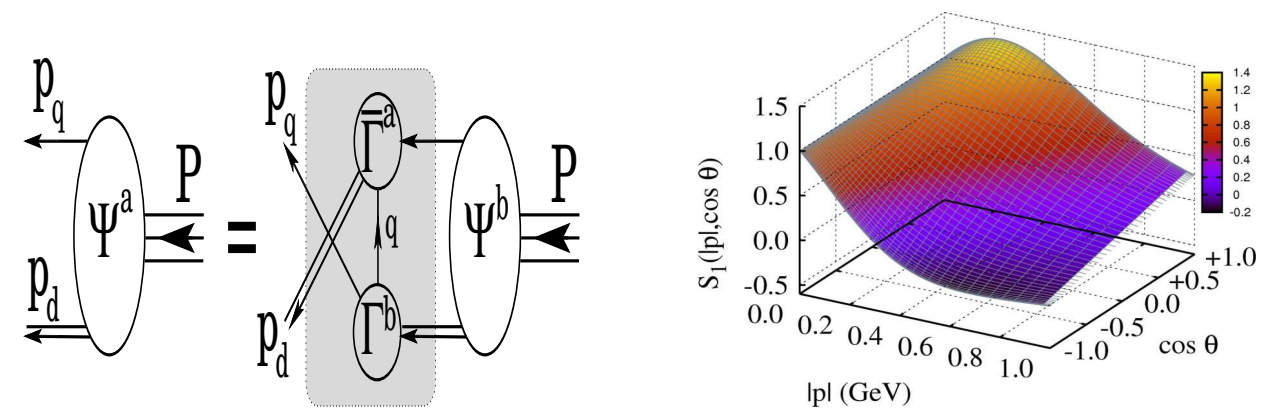

Figure 1. Left panel: Poincaré covariant Faddeev equation. $\Psi$ is the Faddeev amplitude for a baryon of total momentum $P=p_{q}+p_{d}$, where $p_{q, d}$ are, respectively, the momenta of the quark and diquark within the boundstate. The shaded area demarcates the Faddeev equation kernel: single line, dressed-quark propagator; $\Gamma$, diquark correlation amplitude; and double line, diquark propagator. Right panel: Dominant piece in the nucleon's eightcomponent Poincaré-covariant Faddeev amplitude: $S_{1}(|p|, \cos \theta)$. In the nucleon rest frame, this term describes that piece of the quark-diquark relative momentum correlation which possesses zero intrinsic quark-diquark orbital angular momentum, i.e. $L=0$ before the propagator lines are reattached to form the Faddeev wave function. Referring to Fig. $1, p=P / 3-p_{q}$ and $\cos \theta=p \cdot P / \sqrt{p^{2} P^{2}}$. The amplitude is normalised such that its $U_{0}$ Chebyshev moment is unity at $|p|=0$.

which express the strength and range of the correlation and are each bounded below by the partnered meson's mass, may be associated with these diquarks $[1,6,7]: m_{[u d]_{0^{+}}} \approx 0.7-0.8 \mathrm{GeV}, m_{\{u u\}_{1^{+}}} \approx$ $0.9-1.1 \mathrm{GeV}$, with $m_{\{d d\}_{1^{+}}}=m_{\{u d\}_{1^{+}}}=m_{\{u\}_{1^{+}}}$in the isospin symmetric limit. Realistic diquark correlations are also soft. Finally, they possess an electromagnetic size that is bounded below by that of the analogous mesonic system, viz. [8, 9]: $r_{[u d]_{0^{+}}} \gtrsim r_{\pi}, r_{\{u u\}_{1^{+}}} \gtrsim r_{\rho}$, with $r_{\{u u\}_{1^{+}}}>r_{[u d]_{0^{+}}}$.

\section{Diquarks inside the nucleon}

The existence of tight diquark correlations considerably simplifies analyses of the three valence-quark scattering problem and hence baryon bound states. The nucleon is a compound system whose properties and interactions are primarily determined by the quark+diquark structure evident in the Poincaré covariant Faddeev equation depicted in the left panel of Fig. 1. While an explicit three-body term might affect fine details of baryon structure, the dominant effect of non-Abelian multi-gluon vertices is expressed in the formation of diquark correlations.

A nucleon (and kindred baryons) described by the left panel of Fig. 1 can be view as a Borromean bound-state, the binding within which has two contributions. One part is expressed in the formation of tight diquark correlations. That is augmented, however, by attraction generated by the quark exchange depicted in the shaded area of the left panel of Fig. 1. This exchange ensures that diquark correlations within the nucleon are fully dynamical: no quark holds a special place because each one participates in all diquarks to the fullest extent allowed by its quantum numbers. The continual rearrangement of the quarks guarantees, inter alia, that the nucleon's dressed-quark wave function complies with Pauli statistics.

It is important to highlight that both scalar-isoscalar and pseudovector-isotriplet diquark correlations feature within a nucleon. The relative probability of scalar versus pseudovector diquarks in a nucleon is a dynamical statement. Realistic computations predict a scalar diquark strength of approximately $60 \%$ [3-5]. As will become clear, this prediction can be tested by contemporary experiments.

The quark+diquark structure of the nucleon is elucidated in the right panel of Fig. 1, which depicts the leading component of its Faddeev amplitude: with the notation of Ref. [4], $S_{1}(|p|, \cos \theta)$, computed 

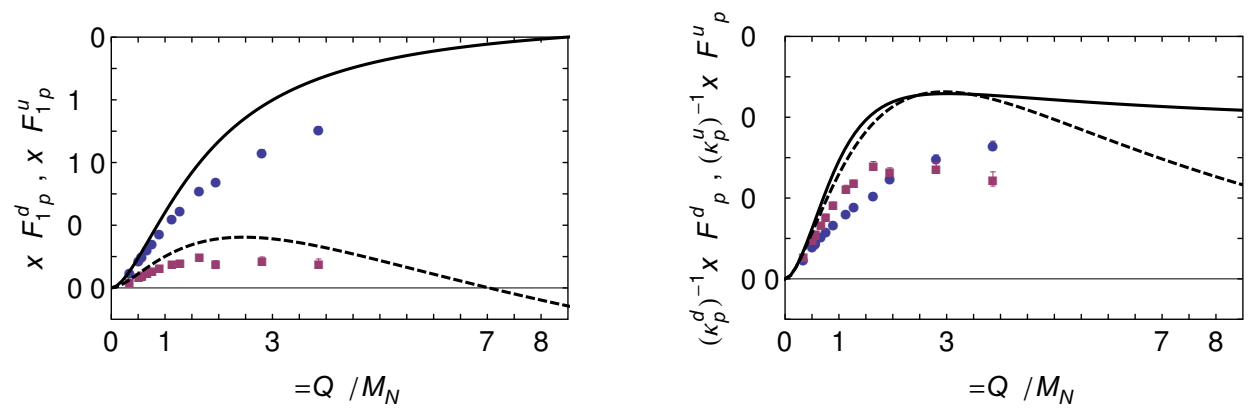

Figure 2. Left panel: Flavour separation of the proton's Dirac form factor as a function of $x=Q^{2} / M_{N}^{2}$. The solid-curve is the $u$-quark contribution, and the dashed-curve is the $d$-quark contribution. Experimental data taken from Ref. [10] and references therein: circles $-u$-quark; and squares $-d$-quark. Right panel: Same for Pauli form factor.

using the Faddeev kernel described therein. This function describes a piece of the quark+scalardiquark relative momentum correlation. Notably, in this solution of a realistic Faddeev equation there is strong variation with respect to both arguments. Support is concentrated in the forward direction, $\cos \theta>0$, so that alignment of $p$ and $P$ is favoured; and the amplitude peaks at $\left(|p| \simeq M_{N} / 6, \cos \theta=1\right)$, whereat $p_{q} \approx P / 2 \approx p_{d}$ and hence the natural relative momentum is zero. In the antiparallel direction, $\cos \theta<0$, support is concentrated at $|p|=0$, i.e. $p_{q} \approx P / 3, p_{d} \approx 2 P / 3$.

\section{Predictions of diquark clustering}

A nucleon described by the Faddeev equation in Fig. 1 is constituted from dressed-quarks, any two of which are always correlated as either a scalar or pseudovector diquark. If this is a veracious description of Nature, then the presence of these correlations must be evident in many physical observables. We focus our attention on the flavour separated versions of the Dirac a Pauli form factors of the nucleon. The evaluation within our framework of the nucleon electromagnetic current is detailed in Ref. [4] and the results we describe herein are derived from that analysis.

Figure 2 displays the proton's flavour separated Dirac and Pauli form factors. The salient features of the data are: the $d$-quark contribution to $F_{1}^{p}$ is far smaller than the $u$-quark contribution; $F_{2}^{d} / \kappa_{d}>$ $F_{2}^{u} / \kappa_{u}$ on $x<2$ but this ordering is reversed on $x>2$; and in both cases the $d$-quark contribution falls dramatically on $x>3$ whereas the $u$-quark contribution remains roughly constant. Our calculations are in semi-quantitative agreement with the empirical data.

It is natural to seek an explanation for the pattern of behaviour in Fig. 2. We have emphasised that the proton contains scalar and pseudovector diquark correlations. The dominant piece of its Faddeev wave function is $u[u d]$; namely, a $u$-quark in tandem with a $[u d]$ scalar correlation, which produces $62 \%$ of the proton's normalisation. If this were the sole component, then photon- $d$-quark interactions within the proton would receive a $1 / x$ suppression on $x>1$, because the $d$-quark is sequestered in a soft correlation, whereas a spectator $u$-quark is always available to participate in a hard interaction. At large $x=Q^{2} / M_{N}^{2}$, therefore, scalar diquark dominance leads one to expect $F^{d} \sim F^{u} / x$. Available data are consistent with this prediction but measurements at $x>4$ are necessary for confirmation.

It is natural now to consider the proton ratio: $R_{21}(x)=x F_{2}(x) / F_{1}(x), x=Q^{2} / M_{N}^{2}$, drawn in Fig. 3 . The momentum dependence of $R_{21}(x)$ is principally determined by the scalar diquark component of the proton. Moreover, the rest-frame $L=1$ terms are seen to be critical in explaining the data: the behaviour of the dashed (green) curve highlights the impact of omitting these components. 


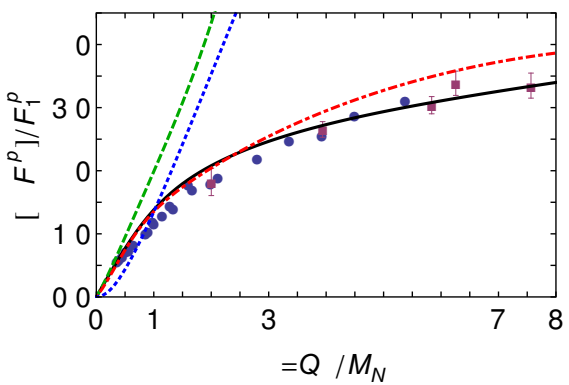

Figure 3. Proton ratio $R_{21}(x)=x F_{2}(x) / F_{1}(x), x=Q^{2} / M_{N}^{2}$. Curves: solid (black) - full result, determined from the complete proton Faddeev wave function and current; dot-dashed (red) - momentum-dependence of the scalar-diquark contribution; dashed (green) - momentum-dependence of that component of the scalar diquark contribution to the proton's Faddeev wave function which is purely $S$-wave in the rest-frame; dotted (blue) - momentum-dependence of the pseudovector diquark contribution. The data are drawn from Ref. [10] and references therein.

\section{Summary}

We have explained that the same interaction which produces mesons is able to generate tight, dynamical colour-antitriplet quark-quark correlations inside baryons. The quark-diquark picture of a baryon should have numerous empirical consequences. Focusing on the flavour-separated proton's Dirac and Pauli form factors, we have seen that dominant scalar diquark correlations inside the proton is enough to observe that, at large $Q^{2}$, the $d$-quark contributions to both Dirac and Pauli proton form factors are reduced relative to the $u$-quark contributions. Moreover, the explained reduction of the ratios $F_{1}^{d} / F_{1}^{u}$ and $F_{2}^{d} / F_{2}^{u}$ at high $Q^{2}$ in the quark-diquark picture are responsible of the precocious scaling of the $F_{2}^{p} / F_{1}^{p}$ observed experimentally.

\section{Acknowledgements}

A significant body of this work is drawn from Ref. [11]. I am grateful for insightful comments from V. Mokeev, I. C. Cloët, R. Gothe, and T.-S.H. Lee. I acknowledges financial support from a postdoctoral IUFFyM contract at Universidad de Salamanca, Spain.

\section{References}

[1] R.T. Cahill, C.D. Roberts, J. Praschifka, Phys. Rev. D36, 2804 (1987)

[2] A. Bender, C.D. Roberts, L. Von Smekal, Phys. Lett. B380, 7 (1996)

[3] I.C. Cloët, G. Eichmann, B. El-Bennich, T. Klahn, C.D. Roberts, Few Body Syst. 46, 1 (2009)

[4] J. Segovia, I.C. Cloët, C.D. Roberts, S.M. Schmidt, Few Body Syst. 55, 1185 (2014)

[5] J. Segovia, B. El-Bennich, E. Rojas, I.C. Cloët, C.D. Roberts, S.S. Xu, H.S. Zong, Phys. Rev. Lett. 115, 171801 (2015)

[6] C. Alexandrou, P. de Forcrand, B. Lucini, Phys. Rev. Lett. 97, 222002 (2006)

[7] R. Babich et al., Phys. Rev. D76, 074021 (2007)

[8] P. Maris, Few Body Syst. 35, 117 (2004)

[9] H.L.L. Roberts et al., Phys. Rev. C83, 065206 (2011)

[10] G.D. Cates, C.W. de Jager, S. Riordan, B. Wojtsekhowski, Phys. Rev. Lett. 106, 252003 (2011)

[11] J. Segovia, C.D. Roberts, S.M. Schmidt, Phys. Lett. B750, 100 (2015) 